\title{
Los modelos de conservación biológica divergente y convergente: Una mirada desde las perspectivas de la ecología del paisaje y la teoría de metapoblaciones
}

\author{
Cristian Kraker-Castañeda ${ }^{1,2^{*}}$, Lorena Soto-Pinto ${ }^{3}$ \\ ${ }^{1}$ Departamento de Conservación de la Biodiversidad, El Colegio de la Frontera Sur (ECOSUR), San Cristóbal de Las Casas, \\ Chiapas, México y ${ }^{2}$ Escuela de Biología, Universidad de San Carlos de Guatemala (EBUSAC), Guatemala. ${ }^{3}$ Departamento de \\ Agricultura, Sociedad y Ambiente, El Colegio de la Frontera Sur (ECOSUR), San Cristóbal de Las Casas, Chiapas, México.
}

*Autor al que se dirige la correspondencia: ckraker@ecosur.edu.mx

Recibido: 02 de Julio 2015 / Revisión: 18 de Agosto 2015 / Aceptado: 15 de Septiembre 2015 / Disponible en línea: 16 de noviembre 2015

\section{Resumen}

\begin{abstract}
T a pérdida de biodiversidad en paisajes agrícolas es un asunto preocupante a nivel mundial y tema central Lde la investigación contemporánea. Este fenómeno puede ser abordado desde dos perspectivas principales: la de la biología de la conservación y la agroecología. La primera enfatiza la importancia de la preservación de los ecosistemas naturales, ya que otros usos del suelo son considerados de legitimidad menor. Para la segunda, el interés se dirige a los agroecosistemas y la biodiversidad es relevante solamente si tiene una conexión con la sostenibilidad de los mismos. La realidad, es que hay agroecosistemas que albergan riqueza en el mismo orden de magnitud que en áreas conservadas y que la pérdida de algunas especies, aparentemente sin valor en la producción del sistema, puede desencadenar efectos cascada si estas son clave en las redes tróficas. Los modelos conceptuales de conservación biológica divergente/convergente, brindan argumentos sobre lo que debería ser la relación entre la agricultura y la biodiversidad; sin embargo, debido a su carga ideológica usualmente derivan en problemas de contextualización. Aquí se refuerzan elementos que parten de la ecología del paisaje y la teoría de metapoblaciones, cuyo soporte proviene de datos empíricos, para repensar este debate con implicaciones para las estrategias de conservación en la región.
\end{abstract}

\begin{abstract}
$\mathrm{B}^{\mathrm{i}}$ iodiversity loss in agricultural landscapes is a global issue of concern, and it is subject of much contemporary research. This phenomenon can be addressed through two main perspectives: conservation biology and agroecology. The first emphasizes the importance of preserving natural ecosystems, considering the study of other land uses to be less important. The second focus is geared towards agro-ecosystems, and biodiversity is considered to be relevant only if it has a connection with sustainability. The reality is that there are agro-ecosystems that can harbor species in the same order of magnitude as in conserved areas, and that the loss of species that have no apparent role in the system's productivity can provoke cascade effects if they are critical in the trophic web. The divergent/ convergent conceptual models of biological conservation provide arguments of what should be the relationship between agriculture and biodiversity; however, their ideological backgrounds usually derive in contextualization problems. Here we reinforce basic elements of landscape ecology and the metapopulation theory both with support in empirical data, as a way of rethink this debate with implications in regional conservation strategies.
\end{abstract}




\section{Introducción}

La pérdida de biodiversidad es un fenómeno alarmante a nivel mundial y actualmente estamos presenciando una tasa de extinción acelerada que por mucho supera la de especiación, y por esto su relevancia. De esta manera nos enfrentamos con una situación concreta, una crisis de la biodiversidad que requiere de disciplinas orientadas a solucionar problemas inmediatos derivados del uso de recursos con las herramientas disponibles (Meffe, Carroll, \& Gromm, 2006). Particularmente, los paisajes agrícolas son escenarios en donde las actividades humanas pueden poner en riesgo a especies cuya capacidad de respuesta es lenta en relación con la velocidad de transformación de su entorno. En estas áreas se han dirigido esfuerzos de investigación para explicar patrones y procesos de la biodiversidad con la finalidad de mejorar las estrategias de conservación biológica, tomando en cuenta la importancia de prácticas agrícolas alternativas.

Los modelos de conservación biológica divergente/convergente brindan argumentos sobre lo que debería ser la relación entre la agricultura y la biodiversidad (Perfecto \& Vandermeer, 2012). El primero, también denominado posición de separación entre producción y conservación, asume que la intensificación agrícola es necesaria para aumentar la productividad y permitir la "liberación de tierras" para su protección (Green, Cornell, Scharlemann, \& Balmford, 2005; Perfecto \& Vandermeer, 2012); en otras palabras, se destinan zonas específicas para el resguardo de la biodiversidad y otras exclusivamente para producción. El segundo, también denominado posición de integración de la producción y conservación, se inclina por prácticas agroecológicas que pueden mantener la biodiversidad a nivel local y a escala de paisaje (Perfecto \& Vandermeer, 2012). Recientemente, se ha reconocido que la ecología del paisaje y la teoría de metapoblaciones, cuyo soporte son datos empíricos, brindan elementos que resuelven mucho del debate sobre este tema (Perfecto \& Vandermeer, 2012; Tscharntke et al., 2012).

\section{Contenido}

\section{Efectos de la transformación del paisaje en la biodiversidad}

La degradación, pérdida y fragmentación de los hábitats se consideran transformaciones de distinta se- veridad y es tema central de mucha de la investigación a nivel mundial (Bennett \& Saunders, 2010; Groom \& Vynne, 2006; Manning, Lindenmayer, \& Nix, 2004). La degradación del hábitat generalmente se refiere a los impactos que afectan a muchas pero no todas las especies, que pueden ser temporales y que no implican la pérdida total de hábitat para todas las especies, más bien representa una reducción en la capacidad de un ecosistema para mantener subconjuntos de especies; en contraste, la pérdida de hábitat se refiere a impactos severos a través de los cuales todas, o casi todas las especies, son afectadas negativamente y los hábitats ya no son capaces de mantener más que una fracción de sus funciones y especies originales (Groom \& Vynne, 2006).

Cuando la fragmentación del hábitat ocurre, surgen tres procesos interrelacionados (Bennett \& Saunders, 2010): (1) la reducción de la cantidad total de vegetación; (2) la subdivisión de la vegetación remanente; y (3) la introducción y reemplazo por nuevos usos del suelo. La fragmentación tiene relación con los patrones y procesos de la biodiversidad, y una forma de contextualizarla es a nivel de paisaje (Fahrig, 2003), es decir en el escenario en el que las poblaciones se desarrollan, cuya escala depende del enfoque y/o la especie en estudio ya que la respuesta/percepción a la distribución espacial de los recursos puede presentarse a nivel de gremios como un reflejo de tendencias adaptativas (e.g. Schnitzler \& Kalko, 2001) o bien ser especie-específica y dependiente de rasgos particulares de historia natural (e.g. Pinto \& Keitt, 2008).

Los patrones de la biodiversidad pueden ser conceptualizados en atributos como los siguientes (Bennett \& Saunders, 2010): (1) la relación especies-área, ya que fragmentos pequeños alojan subconjuntos compuestos por las especies comunes, a lo que denominamos anidamiento; (2) el efecto de borde e interior cuya magnitud está relacionada con el tamaño y forma de los fragmentos, e influye en las condiciones físicas del hábitat y consecuentemente en la ocurrencia de las especies; (3) la conectividad en el paisaje desde la perspectiva de la especie, que es distinta a la del humano; (4) la permeabilidad de la matriz, es decir la facilidad que ofrece para el desplazamiento de especies no típicas. En cambio, los procesos pueden ser exógenos (e.g. cambios en las condiciones físicas del hábitat) o endógenos (e.g. cambios en la conducta de las especies), y determinísticos (e.g. poblaciones pequeñas inducidas) o estocásticos (e.g. poblaciones que por eventos al azar alcanzan un umbral de densidad a partir del cual disminuye su fecundidad y 
supervivencia, a lo que se denomina efecto Allee), y derivar en una mayor susceptibilidad a la extinción (Bennett \& Saunders, 2010; Fischer \& Lindenmayer, 2007). De esta manera, las causas de extinción poblacional pueden ser genéticas, demográficas y/o ambientales, y el nivel de vulnerabilidad depende en gran medida de rasgos ecológicos como el grado de especialización, tolerancia a la perturbación, capacidad de movilidad, entre otros.

\section{Modelos conceptuales en ecología del paisaje}

Los modelos, aunque difieren en sus supuestos, tienen como finalidad proveer un marco teórico para establecer causalidades ecológicas a través de datos empíricos (Fischer \& Lindenmayer, 2007). La búsqueda de la simplificación de los ecosistemas condujo a observaciones como las de Humboldt en el siglo XIX: Áreas más grandes contienen más especies que áreas pequeñas (Seip \& Wenstøp, 2006). Esta generalización, posteriormente desarrollada en lo que conocemos como la función de relación especies-área, es el punto de partida para mucha de la teoría aún vigente; sin embargo, no es sino con el surgimiento de las teorías de biogeografía de islas (TBI) y metapoblaciones (Hanski, 1999; Jules \& Shahani, 2003; Levins, 1969; Vandermeer \& Carvajal, 2001), que el estudio del efecto de la fragmentación de los hábitats en la biodiversidad encuentra sus pilares.

En 1967, MacArthur y Wilson desarrollaron la TBI (también conocida como modelo de islas), cuyas predicciones son simples y elegantes al relacionar el número de especies en una isla con su área y grado de aislamiento: Las islas que son relativamente pequeñas y/o aisladas experimentan una disminución en sus tasas de inmigración, alojan poblaciones con probabilidades de extinción altas $\mathrm{y}$, eventualmente, contendrán un número de especies menor (Bennett \& Saunders, 2010; Fischer \& Lindenmayer, 2007; Franklin \& Lindenmayer, 2009). Este marco teórico, posteriormente trasladado a ecosistemas terrestres, fue fundamento para un grupo de modelos clásicos que incluye el de anidamiento y de parche-matriz-corredor, y permitió estudiar en una analogía cómo en los continentes las actividades humanas crean hábitats o islas (fragmentos o parches relativamente homogéneos), inmersos en una matriz o mar de un hábitat predominante (Forman, 1995; Jules \& Shahani, 2003; Willig, Presley, Bloch, \& Genoways, 2009), que además puede ser atravesado por corredores los cuales son distinguibles de la matriz de fondo (Forman, 1995; Lindenmayer \& Burgman,
2005) y que pueden propiciar conectividad (Diamond, 1975; Forman, 1995).

Alternativamente, surgió el grupo de modelos denominados de continuidad, los cuales emergen como una conceptualización de los paisajes con base en el precepto de la vegetación como un continuo en contraposición a elementos discretos, es decir "un cambio en la composición de especies a través de gradientes ambientales" y representan una antítesis a la teoría que planteaba que las comunidades de plantas son poblaciones formando unidades naturales homogéneas, discretas y reconocibles (Austin, 1985; Fischer \& Lindenmayer, 2006; Manning et al., 2004). El modelo de continuidad más recurrido es el de paisaje variegado (MacIntire \& Barrett, 1992), que reconoce cambios graduales en la cobertura de la vegetación. Este modelo fue desarrollado, en parte, para incorporar gradientes en la calidad de los hábitats (Lindenmayer \& Burgman, 2005; MacIntire \& Barrett, 1992) y enfatiza en las respuestas individuales, es decir en la variedad de formas que los organismos perciben y responden al ambiente (Manning et al., 2004); sin embargo, la falta de datos empíricos apoyando su marco conceptual (Fischer, Lindenmayer, \& Fazey, 2004), así como la necesidad de herramientas de análisis espacial sofisticadas como imágenes de sensores remotos con resolución muy alta para mapear gradientes de recursos (e.g. Price et al., 2009), han tenido como consecuencia que tenga un desarrollo lento (Fischer et al., 2004).

Por otro lado la teoría de metapoblaciones, definidas estas como "colecciones de subpoblaciones que ocupan un determinado porcentaje de hábitats adecuados en el paisaje", surge con implicaciones evidentes, ya que una matriz poco permeable puede representar una barrera física para la movilidad de los organismos en un paisaje fragmentado, con consecuencias demográficas (Levins, 1969; Vandermeer \& Carvajal, 2001). Las metapoblaciones se encuentran vinculadas entre sí por la inmigración y la emigración, y se mantienen por la dispersión desde hábitats fuente, donde el éxito reproductivo (no precisamente la densidad) sobrepasa la mortalidad, hacia hábitats sumidero donde de no existir inmigración periódica, las condiciones de calidad menor favorecerían su desaparición (Dunning, Groom, \& Pulliam, 2006; Pulliam, 1988).

Dos aspectos fundamentales en el criticismo de la TBI son el paradigma parche-matriz y su visión blanco/negro (hábitat y no hábitat) desde una perspectiva humana (Franklin \& Lindenmayer, 2009). Entre sus supuestos la matriz es homogénea y ecológicamente 
irrelevante (Jules \& Shahani, 2003; Vandermeer \& Carvajal, 2001); sin embargo, el modelo de parche-matriz-corredor sigue siendo el background para muchos de los estudios sobre el efecto de la transformación de los paisajes en la biodiversidad. En este sentido, lo que es importante tener en cuenta es que la matriz puede representar en un extremo un espacio inhabitable para algunas especies y en el otro un hábitat continuo semejante a los fragmentos (Vandermeer \& Carvajal, 2001), o bien un punto intermedio como un mosaico altamente heterogéneo a través del cual las especies pueden encontrar condiciones para movilizarse (Forman, 1995; Jules \& Shahani, 2003; Perfecto \& Vandermeer, 2002; Vandermeer \& Carvajal, 2001).

\section{La importancia del paisaje agrícola en la con- servación biológica}

En el contexto de la agroecología surge el concepto de matriz agrícola, que usualmente consiste en diferentes tipos de agroecosistemas; sin embargo, automáticamente se presume tiene una calidad tan baja que es considerada como hábitat sumidero (Perfecto \& Vandermeer, 2002). El tipo de agricultura y su manejo a nivel local (nivel de predio) determinan en gran medida la calidad de la matriz agrícola y su permeabilidad (Perfecto \& Vandermeer, 2012). La calidad de un agroecosistema puede entenderse como el filtro que permite la movilidad de los organismos, considerándose alta cuando presenta pocas barreras (Vandermeer \& Carvajal, 2001).

Asimismo, se menciona que la calidad de la matriz con respecto a los procesos metapoblacionales incrementa con la diversificación de los agroecosistemas (Perfecto \& Vandermeer, 2012). Entonces, los esfuerzos de conservación de la biodiversidad puede que sean más efectivos promoviendo el mejoramiento de la matriz, que por ejemplo planificando corredores "ideales" los cuales en muchos casos se asume deben ser de una calidad semejante a la del mismo fragmento (Perfecto \& Vandermeer, 2002). Por lo tanto, paisajes complejos pueden promover la diversidad local en los mismos agroecosistemas, lo que puede compensar manejos locales altamente intensivos (Tscharntke, Klein, Kruess, Steffan-Dewenter, \& Thies, 2005).

Las variables para medir la calidad de una matriz dependen del enfoque, que puede ser local y de paisaje. A nivel local, es importante evaluar la contribución de las unidades paisajísticas predominantes con base en parámetros como la estructura y la composición de la vegetación y su poder explicativo para la biodiversidad, o incluso variaciones en el régimen de aplicación de agroquímicos (e.g. De la Mora, Murnen, \& Philpott, 2013). Por ejemplo, los ecólogos frecuentemente estudian cronosecuencias de la vegetación, las mismas que muestran diferencias en la comunidad de plantas con relación al estadio de regeneración, con implicaciones en la permeabilidad y disponibilidad de recursos, factores que pueden afectar la movilidad de los organismos (Jules \& Shahani, 2003). A nivel de paisaje, los agroecosistemas que componen la matriz pueden ser clasificados en variables de composición, que son relativas a la ocurrencia y proporción de hábitats, y configuración o arreglo espacial (MacGarigal, Cushman, Neel, \& Ene, 2002); por ejemplo, es posible evaluar la importancia relativa de la distancia entre los agroecosistemas y los fragmentos de vegetación natural para la riqueza y abundancia de especies (e.g. De la Mora et al., 2013).

\section{Los modelos de conservación biológica diver-} gente $\mathbf{y}$ convergente

Los modelos de conservación biológica divergente/convergente son dos argumentos debatidos sobre lo que debería ser la relación entre la agricultura y la biodiversidad, cuyos supuestos algunos son explícitos y otros no (Perfecto \& Vandermeer, 2012), lo que hace de su marco conceptual poco claro (Fischer et al., 2014). Estos modelos contienen una carga ideológica fuerte, lo que deriva muchas veces en problemas de contextualización (Perfecto \& Vandermeer, 2012).

El modelo divergente o land-sparing, argumenta que la intensificación de la agricultura es necesaria para aumentar la productividad (alimento) por área para una población mundial creciente, teniendo como resultado la "liberación de tierras" para la conservación de la biodiversidad (Green et al., 2005; Perfecto \& Vandermeer, 2012); sin embargo, las dos variables consideradas no son totalmente independientes, ya que la biodiversidad vía servicios ecosistémicos puede tener influencia en la productividad (Fischer et al., 2014). Un ejemplo puntual es el estudio de Williams-Guillén, Perfecto y Vandermeer (2008). Estos autores observaron un aumento en la proporción de artrópodos en el follaje de cafetales en una región del Soconusco en Chiapas, México, cuando la actividad depredadora de murciélagos insectívoros que se alimentan de superficies gleaning bats fue interrumpida mediante experimentos de exclusión. Esta interrupción en la red trófica, podría propiciar 
mayor actividad herbívora en los cafetales y consecuentemente riesgos en la producción misma del sistema. Por otro lado, el supuesto implícito de este modelo es la necesidad de sobreproducción de alimento y ya es reconocido que el verdadero problema es la desigualdad en su distribución y accesibilidad, por lo que algunos autores consideran este argumento como una falacia (Perfecto \& Vandermeer, 2012).

Aquellos que promueven la intensificación de la agricultura como una estrategia de conservación además ignoran sus consecuencias negativas, ya que se han documentado esquemas muy industrializados y nefastos para el ambiente (Tscharntke et al., 2012). Asimismo, en el modelo divergente se asume que habrá más disponibilidad de tierras para conservar; sin embargo, los contextos políticos y socioeconómicos que determinan el uso de la tierra son complejos y raramente esto sucede, además que se promueve la implementación de agroquímicos y otros insumos dañinos para el ambiente y el humano (Perfecto \& Vandermeer, 2012). Más aún, para algunos productos agrícolas hay períodos en el que el problema es la sobreproducción: es decir, la oferta es mucho mayor que la demanda y el precio del producto en el mercado está por debajo del de producción (Perfecto \& Vandermeer, 2012).

El modelo convergente o land-sharing, argumenta que la agricultura alternativa, diversa y agroecológica, también denominada orgánica o amigable con el ambiente (Hodgson, Kunin, Thomas, Benton, \& Gabriel, 2010) puede mantener la biodiversidad a nivel local y a escala de paisaje (Green et al., 2005; Perfecto \& Vandermeer, 2012). Esta práctica ha sido considerada como una alternativa que ha favorecido a la producción a pequeña escala, e incorpora nociones de seguridad y soberanía alimentaria, priorizando las economías campesinas (Perfecto \& Vandermeer, 2012).

Una consideración clave según varios autores es el efecto neto de cada modelo en la biodiversidad, tomando en cuenta la productividad de los sistemas (e.g. Chandler et al., 2013; Hodgson et al., 2010). El cultivo de café ha sido uno de los sistemas de producción centrales en la investigación sobre esta temática (Chandler et al., 2013). Este tipo de agroecosistema representa diferentes "diseños biofísicos" y niveles de manipulación de los hábitats (Moguel \& Toledo, 1999), y se presenta en un gradiente continuo desde el manejo tradicional-rústico de calidad alta, al moderno-intensivo de calidad baja (Moguel \& Toledo, 1999; Perfecto, Rice, Greenberg, \& Van der Voort, 1996; Perfecto \& Vandermeer, 2002). Hay estudios que demuestran que en este tipo de agroecosistemas, la riqueza y abundancia de especies para varios grupos taxonómicos tiene una similitud alta con los ecosistemas nativos (e.g. Greenberg, Bichier, Angon, \& Reitsma, 1997; Pineda, Moreno, Escobar, \& Halffter, 2005; Williams-Guillén \& Perfecto, 2010). Por ejemplo, Numa, Verdú y Sánchez-Palomino (2005), en su área de estudio, calcularon un recambio bajo de especies de murciélagos filostómidos entre bosques remanentes en una matriz con predominancia de agroecosistemas diversificados, frente a una matriz con agroecosistemas convencionales; estos autores interpretaron dicho resultado como una continuidad mayor en la matriz en el área con manejo agroecológico.

Sin embargo, también hay evidencia que los agroecosistemas pueden ser menos productivos y por lo tanto son poco elegidos en algunas regiones agrícolas (Chandler et al., 2013; Moguel \& Toledo, 1999); además, pueden alojar especies generalistas mientras que las especialistas siguen siendo dependientes del ecosistema nativo (Chandler et al., 2013). En este sentido, la escala es una consideración importante en los sistemas de producción, por lo que en muchos casos no es posible comparar y generalizar resultados de unos cuantos estudios. El modelo divergente frecuentemente asume extensiones geográficas amplias y de grano grueso, mientras que el modelo convergente se enfoca típicamente a integrar la producción y la conservación en una escala menor (Egan \& Mortensen, 2012; Hodgson et al., 2010).

En la actualidad, los casos exitosos de modelos de conservación divergentes son escasos (e.g. Chandler et al., 2013) en comparación con manejos agroecológicos (e.g. Greenberg et al., 1997; Pineda et al., 2005; Williams-Guillén \& Perfecto, 2010); sin embargo, se requiere un esfuerzo mayor de estudio para brindar soporte a las distintas estrategias de conservación biológica. Lo que resulta evidente, es que algunos de los supuestos de estos modelos no son claros e ignoran mecanismos que mantienen a la biodiversidad a nivel de paisaje, lo que puede derivar en extinciones locales y efectos en la misma productividad de los sistemas agrícolas.

\section{Conclusión}

Para algunos autores el debate de si las prácticas agrícolas deben ser intensificadas para incrementar la productividad y así resguardar la biodiversidad en zonas específicas, o si deben ser favorecidas las prácticas 
diversificadas para mantener la biodiversidad en una dinámica con el paisaje agrícola, ha fallado en su implementación efectiva. En la literatura se plantea que la mejor estrategia para la conservación de la biodiversidad manteniendo la producción en un nivel deseado es dependiente del contexto de cada región y de los supuestos de cada modelo. Una de las estrategias propuestas es asegurar la conectividad y migración entre metapoblaciones, a través de una matriz agrícola de calidad alta, considerando además la relevancia de los corredores de vegetación natural y los stepping stones (Perfecto \& Vandermeer, 2012; Tscharntke et al., 2012). Algunos de los supuestos de los modelos de conservación biológica convergente/divergente acarrean problemas de contextualización y consecuentemente derivan en retrocesos en las estrategias de conservación; sin embargo, la ecología del paisaje y la teoría de metapoblaciones brindan elementos para repensar este tema, con un soporte en datos empíricos.

\section{Referencias}

Austin, M. P. (1985). Continuum concept, ordination methods, and niche theory. Annual Review of Ecology and Systematics, 16, 39-61. doi: 10.1146/ annurev.es.16.110185.000351

Bennett, A. F., \& Saunders, D. A. (2010). Habitat fragmentation and landscape change. In N. S. Sodhi \& P. R. Ehrlich (Eds.), Conservation biology for all (Chap. 5, pp. 88-106). New York: Oxford University Press.

Chandler, R. B., King, D. I., Raudales, R., Trubey, R., Chandler, C., \& Arce, V. J. (2013). A small-scale land-sparing approach to conserving biological diversity in tropical agricultural landscapes. Conservation Biology, 27(4), 785-795. doi: 10.1111/ cobi. 12046

De la Mora, A., Murnen, C. J., \& Philpott, S. M. (2013). Local and landscape drivers of biodiversity of four groups of ants in coffee landscapes. Biodiversity and Conservation, 22(4), 871-888. doi: 10.1007/ s10531-013-0454-z

Diamond, J. M. (1975). The island dilemma: Lessons of modern biogeographic studies for the design of natural reserves. Biological Conservation, 7(2), 129-145. doi: 10.1016/0006-3207(75)90052-X
Dunning, J. B., Groom, M. J., \& Pulliam, H. R. (2006). Species and landscape approaches to conservation. En M. J. Groom, G. K. Meffe \& C. R. Carroll (Eds.), Principles of Conservation Biology ( $3^{\text {rd }}$. ed., Chap. 12, pp. 419-465). Sunderland, Massachusets: Sinauer Associates.

Egan, J. F., \& Mortensen, D. A. (2012). A comparison of land-sharing and land-sparing strategies for plant richness conservation in agricultural landscapes. Ecological Applications, 22(2), 459471. doi: 10.1890/11-0206.1

Fahrig, L. (2003). Effects of habitat fragmentation on biodiversity. Annual Review of Ecology, Evolution and Systematics, 34, 487-515. doi: 10.1146/ annurev.ecolsys.34.011802.132419

Fischer, J., Abson, D. J., Butsic, V., Chappell, M. J., Ekroos, J., Hanspach, J., ... von Wehrden, H. (2014). Land sparing versus land sharing: Moving forward. Conservation Letters, 7(3), 149-157. doi: 10.1111/conl.12084

Fischer, J., Lindenmayer, D. B., \& Fazey, I. (2004). Appreciating ecological complexity: Habitat contours as a conceptual landscape model. Conservation Biology, 18(5), 1245-1253. doi: 10.1111/j.1523-1739.2004.00263.x

Fischer, J., \& Lindenmayer, D. B. (2006). Beyond fragmentation: The continuum model for fauna research and conservation in humanmodified landscapes. Oikos, 112(2), 473-480. doi: 10.1111/j.0030-1299.2006.14148.x

Fischer, J., \& Lindenmayer, D. B. (2007). Landscape modification and habitat fragmentation: A synthesis. Global Ecology and Biogeography, 16(3), 265280. doi: 10.1111/j.1466-8238.2007.00287.x

Forman, R. T. (1995). Land mosaics: The ecology of landscapes and regions. New York, Cambridge, United Kingdom: Cambridge University Press.

Franklin, J. F., \& Lindenmayer, D. B. (2009). Importance of matrix habitats in maintaining biological diversity. Proceedings of the National Academy of Science, 106(2), 349-350. doi: 10.1073/pnas.0812016105

Green, R. E., Cornell, S. J., Scharlemann, J. P., \& Balmford, A. (2005). Farming and the fate of wild nature. Science, 307(5709), 550-555. doi: 10.1126/science.1106049 
Greenberg, R., Bichier, P., Angon, A. C., \& Reitsma, R. (1997). Bird populations in shade and sun coffee in central Guatemala. Conservation Biology, 11(2), 448-459. doi: 10.1046/j.15231739.1997.95464.x

Groom, M. J., \& Vynne, C. H. (2006). Habitat degradation and loss. En M. J. Groom, G. K. Meffe \& C. R. Carroll (Eds.), Principles of Conservation Biology $\left(3^{\text {rd }}\right.$. ed., Chap. 6, pp. 173-212). Sunderland, Massachusets: Sinauer Associates.

Hanski, I. (1999). Habitat connectivity, habitat continuity, and metapopulations in dynamic landscapes. Oikos, 87(2), 209-219. doi: 10.2307/3546736

Hodgson, J. A., Kunin, W. E., Thomas, C. D., Benton, T. G., \& Gabriel, D. (2010). Comparing organic farming and land sparing: Optimizing yield and butterfly populations at a landscape level. Ecology Letters, 13(11), 1358-1367. doi: 10.1111/j.14610248.2010.01528.x

Jules, E. S., \& Shahani, P. (2003). A broader ecological context to the fragmentation frenzy: Why matrix habitat is more important than we thought. Journal of Vegetation Science, 14(3), 459-464. doi: 10.1111/j.1654-1103.2003.tb02172.x

Levins, R. (1969). Some demographic and genetic consequences of environmental heterogeneity for biological control. Bulletin of the Entomological Society of America, 15(3), 237-240. doi: dx.doi. org/10.1093/besa/15.3.237

Lindenmayer, D., \& Burgman, M. (2005). Practical conservation biology. Collingwood, Australia: CSIRO Publishing.

MacIntire, S., \& Barrett, G. W. (1992). Habitat variegation, an alternative to fragmentation. Conservation Biology, 6(1), 146-147. doi: 10.1046/j.15231739.1992.610146.x

MacGarigal, K., Cushman, S. A., Neel, M. C., \& Ene, E. (2002). FragStats: Spatial pattern analysis program for categorical maps. [software]. Amherst: University of Massachusets.

Manning, A. D., Lindenmayer, D. B., \& Nix, H. A. (2004). Continua and umwelt: Novel perspectives on viewing landscapes. Oikos, 104(3), 621-628. doi: 10.1111/j.0030-1299.2004.12813.x
Meffe, G. K., Carroll, C. R., \& Groom, M. J. (2006). What is Conservation Biology? En M. J. Groom, G. K. Meffe \& C. R. Carroll (Eds.), Principles of Conservation Biology ( $3^{\text {rd }}$. ed. Chap. 1, pp. 3-25). Sunderland, Massachusets: Sinauer Associates.

Moguel, P., \& Toledo, V. M. (1999). Biodiversity conservation in traditional coffee systems of Mexico. Conservation Biology, 13(1), 11-21. doi: 10.1046/j.1523-1739.1999.97153.x

Numa, C., Verdú, J. R., \& Sánchez-Palomino, P. (2005). Phyllostomid bat diversity in a variegated coffee landscape. Biological Conservation, 122, 151158. doi: 10.1016/j.biocon.2004.07.013

Perfecto, I., Rice, R. A., Greenberg, R., \& Van der Voort, M. E. (1996). Shade coffee: A disappearing refuge for biodiversity. BioScience, 46(8), 598608. doi: $10.2307 / 1312989$

Perfecto, I., \& Vandermeer, J. (2002). Quality of agroecological matrix in a tropical montane landscape: Ants in coffee plantations in southern Mexico. Conservation Biology, 16(1), 174-182. doi: 10.1046/j.1523-1739.2002.99536.x

Perfecto, I., \& Vandermeer, J. (2012). Separación o integración para la conservación de biodiversidad: La ideología detrás del debate "land-sharing" frente a "land-sparing". Ecosistemas, 21(1-2), 180-191.

Pineda, E., Moreno, C., Escobar, F., \& Halffter, G. (2005). Frog, bat and dung beetle diversity in the cloud forest and coffee agroecosystems of Veracruz, Mexico. Conservation Biology, 19(2), 400-410. doi: 10.1111/j.1523-1739.2005.00531.x

Pinto, N., \& Keitt, T. H. (2008). Scale-dependent responses to forest cover displayed by frugivore bats. Oikos, 117(11), 1725-1731. doi: 10.1111/j.16000706.2008.16495.x

Price, B., McAlpine, C. A., Kutt, A. S., Phinn, S. R., Pullar, D. V., \& Ludwig, J. A. (2009). Continuum or discrete patch landscape models for savanna birds? Towards a pluralistic approach. Ecography, 32(5), 745-756. doi: 10.1111/j.16000587.2009.05670.x

Pulliam, H. R. (1988). Sources, sinks, and population regulation. American Naturalist, 132(5), 652-661. doi: $10.1086 / 284880$

Schnitzler, H.-U., \& Kalko, E. K. (2001). Echolocation by insect-eating bats. BioScience, 51(7), 557-569. doi: 10.1641/0006-3568(2001)051[0557 
Seip, K. L., \& Wenstøp, F. (2006). A primer on environmental decision-making. An integrative quantitative approach. Dordrecht, The Netherlands: Springer.

Tscharntke, T., Clough, Y., Wanger, T. C., Jackson, L., Motzke, I., Perfecto, I., ... Whitbread, A. (2012). Global food security, biodiversity conservation and the future of agricultural intensification. Biological Conservation, 151(1), 53-59. doi: 10.1016/j. biocon.2012.01.068

Tscharntke, T., Klein, A. M., Kruess, A., Steffan-Dewenter, I., \& Thies, C. (2005). Landscape perspectives on agricultural intensification and biodiversity ecosystem management. Ecology Letters, 8(8), 857-874. doi: 10.1111/j.1461-0248.2005.00782.x

Williams-Guillén, K., \& Perfecto, I. (2010). Effects of agricultural intensification on the assemblage of leaf-nosed bats (Phyllostomidae) in a coffee landscape in Chiapas, Mexico. Biotropica, 42(5), 605-613. doi: 10.1111/j.1744-7429.2010.00626.x

Williams-Guillén, K., Perfecto, I., \& Vandermeer, J. (2008). Bats limit insects in a neotropical agroforestry system. Science, 320(5872), 70. doi: 10.1126/science.1152944

Willig, M. R., Presley, S. J., Bloch, C. P., \& Genoways, H. H. (2009). Macroecology of Caribbean bats: Effects of area, elevation, latitude, and hurricane-induced disturbance. En T. H. Fleming \& P. A. Racey (Eds.), Island bats. Evolution ecology and conservation (Chap. 8, pp. 216-264). Chicago: The University of Chicago Press.

Vandermeer, J., \& Carvajal, R. (2001). Metapopulation dynamics and the quality of the matrix. American Naturalist, 158(3), 211-220. doi: 10.1086/321318 